International Electronic Journal of Algebra

Volume 26 (2019) 111-121

DOI: $10.24330 /$ ieja. 587002

\title{
A NOTE ON AUTOMORPHISM LIFTABLE MODULES
}

\author{
S. Santhakumar \\ Received: 2 December 2018; Revised: 7 January 2019; Accepted: 10 January 2019 \\ Communicated by Burcu Üngör

\begin{abstract}
A module $M$ is said to be an automorphism liftable module if be lifted to an endomorphism of $M$. In this work, some properties of auto-
\end{abstract} \\ for each submodule $N$ of $M$, every automorphism of the quotient $M / N$ can \\ morphism liftable modules are investigated. Also, characterization for some \\ special rings such as perfect, semiperfect and uniserial are given by using au- \\ tomorphism liftable modules.
}

Mathematics Subject Classification (2010): 16D40, 16W20, 20K10

Keywords: Automorphism liftable module, dual automorphism-invariant module, perfect ring

\section{Introduction and basic definitions}

Throughout this paper, $R$ denotes an associative ring with identity and all modules are assumed to be unital right $R$-modules unless otherwise stated. For any module $M, J(M)$ and $E(M)$ denote the Jacobson radical and injective hull of $M$.

Singh and Srivastava [13], introduced a new class of modules namely dual automorphism invariant modules, which is the dual notion of automorphism invariant modules introduced by Lee and Zhou [9]. Further study of such modules was carried out by various authors in various articles $[1,8,11]$.

A module $M$ is called an automorphism-invariant module [9] if $M$ is invariant under any automorphism of its injective hull, i. e., for any automorphism $\sigma$ of $E(M), \sigma(M) \subseteq M$. In other-hand, $M$ is called an automorphism invariant module if every isomorphism between two essential submodules of $M$ extends to an automorphism of $M$. Clearly, any quasi injective or pseudo injective module is an automorphism-invariant module.

A submodule $N$ of a module $M$ is said to be small in $M$ if there is no proper submodule $K$ of $M$ such that $N+K=M$. The notation $N \ll M$ means that $N$ is small in $M$. A projective cover of a module $M$ is an epimorphism $f: P \rightarrow M$ with $\operatorname{Ker}(f) \ll P$ and $P$ is projective. 
A module $M$ is said to be quasi projective if for any module $A$, epimorphism $q: M \rightarrow A$ and homomorphism $f: M \rightarrow A$, there is an endomorphism $f^{\prime}$ of $M$ such that the diagram

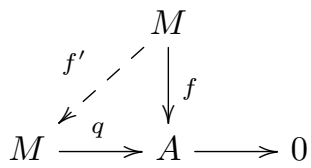

is commutative, that is, $q f^{\prime}=f$.

A module $M$ is called a dual automorphism-invariant module if whenever $K_{1}$ and $K_{2}$ are small submodules of $M$, then any epimorphism $\eta: M / K_{1} \rightarrow M / K_{2}$ with small kernel lifts to an endomorphism $\phi$ of $M$.

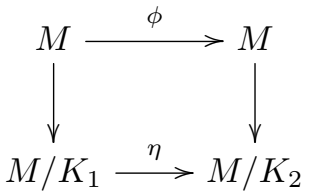

In fact, the lifting $\phi$ is an isomorphism [13]. Every semisimple module is a dual automorphism-invariant module. Quasi projective and pseudo projective modules are dual automorphism-invariant modules. Also, the Prüfer group $\mathbb{Z}\left(p^{\infty}\right)$ is not a dual automorphism invariant module over the ring of integers [13].

In [14], Tuganbaev introduced a new notion namely automorphism-extendable module. A right module $M$ is said to be an automorphism-extendable module if for each submodule $N$ of $M$, every automorphism of the module $N$ can be extended to an endomorphism of $M$. In [14], he proved that every automorphism-invariant module is an automorphism-extendable module. But the converse need not be true. For example, $\mathbb{Z}$ is an automorphism-extendable $\mathbb{Z}$-module but it is not an automorphism-invariant $\mathbb{Z}$-module [14].

The dual notion of automorphism-extendable module was introduced by Selvaraj and Santhakumar in [12]. Such modules were called as automorphism liftable modules. Also, they characterized some rings by the properties of automorphism liftable modules and they studied automorphism liftable modules with the summand sum and summand intersection properties. At the same time Abyzov and Truong [2] introduced the same concept in the name of dual automorphism-extendable module. They proved that dual automorphism-extendable module satisfies the D3 condition. Also, in [1] Abyzov et al. studied the notion of dual strictly automorphismextendable modules which can be viewed as a strongest notion of automorphism liftable modules. They proved that over a perfect ring a module is dual strictly automorphism-extendable if and only if it is dual automorphism-invariant. 
A module $M$ is a Hopf module provided that every surjective endomorphism of $M$ is an automorphism. A submodule $K$ of $M$ is said to be weak supplement of $M$ if there is a submodule $N$ of $M$ such that $N+K=M$ and $N \cap K \ll M$. $M$ is a weakly supplemented module if every submodule of $M$ has a weak supplement.

In this paper, we discuss the question "When a direct sum of automorphism liftable modules will become automorphism liftable?". Also we introduce automorphism liftable cover to characterize some rings.

\section{Automorphism liftable modules}

Selvaraj and Santhakumar introduced the notion automorphism liftable module in [12], and discussed some basic properties of the same. Here we discuss some more basic properties of automorphism liftable modules.

The definition of automorphism liftable module as defined in [12] is

Definition 2.1. [12] A module $M$ is said to be an automorphism liftable module if for each submodule $N$ of $M$, every automorphism of the quotient $M / N$ can be lifted to an endomorphism of $M$.

\section{Example 2.2.}

(1) It is very clear that every quasi (pseudo) projective module is automorphism liftable.

(2) Let $R=\left(\begin{array}{cc}\mathbb{F}_{2} & \mathbb{F}_{2} \\ 0 & \mathbb{F}_{2}\end{array}\right)$ be a ring and $M=\left(\begin{array}{cc}\mathbb{F}_{2} & \mathbb{F}_{2} \\ 0 & 0\end{array}\right)$ be a right $R$ module. Then the only proper subset of $M$ is $N=\left\{\left(\begin{array}{cc}a & a \\ 0 & 0\end{array}\right) \mid a \in \mathbb{F}_{2}\right\}$. Then $M / N$ isomorphic to $\mathbb{F}_{2}$. Hence the only automorphism of $M / N$ is the identity. Therefore $M$ is an automorphism liftable module over the ring $R$.

(3) A weakly supplemented dual automorphism invariant module is an automorphism liftable module $[1,12]$.

(4) Over a perfect ring every flat module is an automorphism liftable module $[12]$.

(5) Over a quasi-Frobenius ring every injective module is an automorphism liftable module [12].

We know that if any two modules $\mathrm{M}$ and $\mathrm{N}$ with projective covers are isomorphic, then their projective covers are isomorphic. The converse part need not be true for all modules. However, here we try to solve the converse with certain condition by using automorphism liftable modules. 
Proposition 2.3. Suppose $P_{1} \stackrel{\phi_{1}}{\longrightarrow} M_{1} \rightarrow 0$ and $P_{2} \stackrel{\phi_{2}}{\longrightarrow} M_{2} \rightarrow 0$ are projective covers of modules $M_{1}$ and $M_{2}$, respectively and $P_{1} \cong P_{2}$ with $M_{1} \oplus M_{2}$ is an automorphism liftable module. Then $M_{1} \cong M_{2}$.

Proof. Since $M_{1}$ and $M_{2}$ have projective covers, $M_{1} \oplus M_{2}$ has a projective cover $P_{1} \oplus P_{2} \stackrel{\phi_{1} \oplus \phi_{2}}{\longrightarrow} M_{1} \oplus M_{2} \rightarrow 0$. Let $\rho_{1}: P_{1} \rightarrow P_{2}$ be an automorphism and $\rho_{2}: P_{2} \rightarrow$ $P_{1}$ be the inverse of $\rho_{1}$. Consider a homomorphism $\rho=\rho_{1} \oplus \rho_{2}: P_{1} \oplus P_{2} \rightarrow P_{1} \oplus P_{2}$, which is an automorphism of $P_{1} \oplus P_{2}$. Since $J\left(P_{1} \oplus P_{2}\right)=J\left(P_{1}\right) \oplus J\left(P_{2}\right)$ is invariant under $\rho$, there exists an automorphism $\widehat{\rho}:\left(P_{1} \oplus P_{2}\right) / J\left(P_{1} \oplus P_{2}\right) \rightarrow\left(P_{1} \oplus P_{2}\right) / J\left(P_{1} \oplus\right.$ $\left.P_{2}\right)$. Also, $\left(P_{1} \oplus P_{2}\right) / J\left(P_{1} \oplus P_{2}\right) \cong\left(P_{1} / J\left(P_{1}\right)\right) \oplus\left(P_{2} / J\left(P_{2}\right)\right)$. Take $J_{1}=\phi_{1}\left(J\left(P_{1}\right)\right)$ and $J_{2}=\phi_{2}\left(J\left(P_{2}\right)\right)$. Since $J\left(P_{1}\right)$ and $J\left(P_{2}\right)$ are small sumodules of $P_{1}$ and $P_{2}$ respectively, clearly, $J_{1}$ and $J_{2}$ are small submodules of $M_{1}$ and $M_{2}$ respectively. Also, $\left(P_{1} / J\left(P_{1}\right)\right) \oplus\left(P_{2} / J\left(P_{2}\right)\right) \cong\left(M_{1} / J_{1}\right) \oplus\left(M_{2} / J_{2}\right) \cong\left(M_{1} \oplus M_{2}\right) /\left(J_{1} \oplus J_{2}\right)$. Then the automorphism $\rho=\rho_{1} \oplus \rho_{2}$ yields an automorphism $\delta=\delta_{1} \oplus \delta_{2}, \delta_{1}$ : $M_{1} / J_{1} \rightarrow M_{2} / J_{2}$ and $\delta_{2}: M_{2} / J_{2} \rightarrow M_{1} / J_{1}$ are isomorphism yielded by $\rho_{1}$ and $\rho_{2}$ respectively.

Since $M_{1} \oplus M_{2}$ is automorphism liftable, $\exists \sigma: M_{1} \oplus M_{2} \rightarrow M_{1} \oplus M_{2}$ is a lifting of $\delta$. Consider the map $f=I_{1} \circ \sigma \circ n_{2}: M_{1} \rightarrow M_{2}$, where $I_{1}: M_{1} \rightarrow M_{1} \oplus M_{2}$ is natural inclusion map and $n_{2}: M_{1} \oplus M_{2} \rightarrow M_{2}$ is natural projection. Then we have $f\left(M_{1}\right)+J_{2}=M_{2}$ and $J\left(M_{2}\right)$ is small in $M_{2}, f\left(M_{1}\right)=M_{2}$, i.e. $f$ is epic. By [12, Corollary 2.10], $f$ is a splitting epimorphism. This implies that $f$ is an isomorphism. This completes the proof.

The direct sum of two automorphism liftable modules need not be automorphism liftable. For example [12], $\mathbb{Z}_{2}$ and $\mathbb{Z}_{4}$ are automorphism liftable $\mathbb{Z}$-modules. But, $\mathbb{Z}_{2} \oplus \mathbb{Z}_{4}$ is not an automorphism liftable $\mathbb{Z}$-module. This motivates us to rise the question "When a direct sum of automorphism liftable modules will become automorphism liftable?". Through following results we partially answer the above question.

Theorem 2.4. Let $P$ be a projective simple module, and $M$ be any automorphism liftable module such that $\operatorname{Hom}(M / K, P)=0$ for any submodule $K$ of $M$. Then $P \oplus M$ is automorphism liftable.

Proof. Set $N=P \oplus M$. Let $K$ be a submodule of $N$, then either $P \subseteq K$ or $P \cap K=0$.

Case 1: Suppose $P \subseteq K, N / K=M / K_{1}$ for some submodule $K_{1} \subseteq M$. Let $f: N / K \rightarrow N / K$ be an automorphism, then we can inherit an automorphism $f_{1}: M / K_{1} \rightarrow M / K_{1}$ from $f$. Since $M$ is automorphism liftable, $\exists g_{1}: M \rightarrow M$ is 
liftable of $f_{1}$. Let $g=g_{1} \oplus I_{P}, I_{P}$ is an identity map of $P$. Then $g: N \rightarrow N$ is a lifting of $f$.

Case 2: Suppose $P \cap K=0, N / K \cong P \oplus M / K_{1}$ for some submodule $K_{1} \subseteq$ $M$. Let $f: N / K \rightarrow N / K$ be an automorphism. Now $f$ can be viewed as $f=$ $\left(\begin{array}{ll}f_{11} & f_{12} \\ f_{21} & f_{22}\end{array}\right)$, where $f_{11}: P \rightarrow P, f_{12}: P \rightarrow M / K, f_{21}: M / K \rightarrow P$ and $f_{22}: M / K \rightarrow M / K$. By hypothesis $f_{21}=0$. Then $f=\left(\begin{array}{cc}f_{11} & f_{12} \\ 0 & f_{22}\end{array}\right)$. Since $f$ is an automorphism, $\exists g=\left(\begin{array}{cc}g_{11} & g_{12} \\ 0 & g_{22}\end{array}\right)$ such that $f g=I=g f$.

$$
\begin{aligned}
f g & = \\
\left(\begin{array}{cc}
f_{11} & f_{12} \\
0 & f_{22}
\end{array}\right)\left(\begin{array}{cc}
g_{11} & g_{12} \\
0 & g_{22}
\end{array}\right) & =\left(\begin{array}{ll}
1 & 0 \\
0 & 1
\end{array}\right) . \\
\left(\begin{array}{cc}
f_{11} g_{11} & f_{11} g_{12}+f_{12} g_{22} \\
0 & f_{22} g_{22}
\end{array}\right) & =\left(\begin{array}{ll}
1 & 0 \\
0 & 1
\end{array}\right) .
\end{aligned}
$$

This implies that $f_{11} g_{11}=I$ and $f_{22} g_{22}=I$. Similarly, we have $g_{11} f_{11}=I$ and $g_{22} f_{22}=I$. Hence $f_{22}$ is an isomorphism of $M / K$. Since $M$ is automorphism liftable, $\exists h_{22}: M \rightarrow M$ is a lifting of $f_{22}$. By the projectivity of $P, \exists h_{12}: P \rightarrow M$ is a lifting of $f_{12}$. Take $h_{11}=f_{11}$ and $h_{21}=0$.

Let $h=\left(\begin{array}{cc}h_{11} & h_{12} \\ 0 & h_{22}\end{array}\right)$. It is easy to check that $h$ is a lifting of $f$. Hence $P \oplus M$ is an automorphism liftable module.

Theorem 2.5. If $M_{i}(i \in I)$ are automorphism liftable modules such that for every submodule $N$ of the direct sum $M=\oplus M_{i}, N_{i}=\oplus\left(N \cap M_{i}\right)$ holds, then $M$ is again an automorphism liftable module.

Proof. Since every submodule $N$ of the direct sum $M=\oplus M_{i}$, holds $N_{i}=\oplus(N \cap$ $\left.M_{i}\right)$, every quotient module $M / N$ of $M$ is of the form $\oplus\left(M / N_{i}\right)$ with $N_{i} \subseteq M_{i}$. By the proof of $\left[4\right.$, Lemma 3], every homomorphism $M_{i} \rightarrow M_{j} / N_{j}$ is trivial. Let $f: M_{i} / N_{i} \rightarrow M_{j} / N_{j}$ be a homomorphism, $g=f \circ n$, where $n: M_{i} \rightarrow M_{i} / N_{i}$ is natural projection, is a homomorphism from $M_{i} \rightarrow M_{j} / N_{j}$. Hence $g=f \circ n=0$ implies $f=0$. Therefore any automorphism $\sigma: M / N \rightarrow M / N$ is a coordinate wise automorphism. This implies that $M$ is an automorphism liftable module.

Proposition 2.6. If $M_{i}(i \in I)$ are quasi projective modules and $M=\oplus M_{i}$ is an automorphism liftable module, then $M$ is quasi projective. 
Proof. The proof is followed by [12, Theorem 2.7].

\section{Automorphism liftable cover}

In this section, we introduce an analog notion of dual automorphism invariant cover, namely automorphism liftable cover via AL-stable submodule. We can view it as a generalization of the quasi projective cover.

Definition 3.1. A submodule $Q$ of $M$ is said to be $A L$-stable if for any submodule $N$ containing $Q$ and automorphism $g: M / N \rightarrow M / N$ having lifting $f \in E n d M$ of $g, f(Q) \subseteq Q$.

Clearly, every invariant submodule of $M$ is an AL-stable submodule of $M$. A zero module is trivially an AL-stable submodule of any module.

The following lemma shows the purpose of defining the notion AL-stable submodule.

Lemma 3.2. If $Q$ is an AL-stable submodule of an automorphism liftable module $M$, then the quotient module $M / Q$ is an automorphism liftable module.

Proof. Let $f:(M / Q) /(N / Q) \rightarrow(M / Q) /(N / Q)$ be an automorphism, where $N$ is a submodule of $M$. Equivalently, $f: M / N \rightarrow M / N$ is an automorphism. Then it can be extended to $g: M \rightarrow M$, and also $Q$ is an AL-stable submodule. Hence $g_{1}: M / Q \rightarrow M / Q$ defined by $g_{1}(m+Q)=g(m)+Q$ is a lifting of $f$.

Lemma 3.3. Let $Q$ be an AL-stable submodule of an automorphism liftable module $M$, and $N$ be a submodule of $M$ such that $Q \subseteq N$. If $N$ is not an AL-stable submodule of $M$, then $N / Q$ is not an $A L$-stable submodule of $M / Q$.

Proof. If $N$ is not an AL-stable submodule of $M$, then there exist submodules $N_{1}$ containing $N$, isomorphism $f: M / N_{1} \rightarrow M / N_{1}$ having lift $f_{1}: M \rightarrow M$ such that $f_{1}\left(n_{0}\right) \notin N$ for some $n_{0} \in N$. Define $g: M / Q \rightarrow M / Q$ by $g(m+Q)=f_{1}(m)+Q$. Then $g\left(n_{0}+Q\right)=f_{1}\left(n_{0}\right)+Q \notin N / Q$. Hence $N / Q$ is not an AL-stable submodule of $M / Q$.

Definition 3.4. An automorphism liftable module $L$ is said to be an automorphism liftable cover of a module $M$ if

(1) there exists a minimal epimorphism $\psi: L \rightarrow M$, i.e. $\operatorname{Ker}(\psi)$ is small.

(2) $\operatorname{Ker}(\psi)$ does not contain any non-zero AL-stable submodule of $L$.

The following theorem gives an existence of an automorphism liftable cover. 
Theorem 3.5. If a module $M$ has a quasi-projective cover, then it has an automorphism liftable cover.

Proof. Let $f: Q \rightarrow M$ be a quasi projective cover of $M$. Let $A$ be a maximal AL-stable submodule of $Q$ contained in $\operatorname{Ker}(f)$. Then by Lemma 3.2, $Q / A$ is an automorphism liftable module. Since $A \subseteq \operatorname{Ker}(f)$, there exists $g: Q / A \rightarrow M$ such that $f=g \circ h$ where $h: Q \rightarrow Q / A$ is a natural homomorphism. Since $h$ is onto, $f$ is onto and $\operatorname{Ker}(g)=\operatorname{Ker}(f) / A$.

Suppose $(B / A)+K \operatorname{Ker}(g)=Q / T$. Then $B+K \operatorname{Ker}(f)=Q$ and so $B=Q$. Therefore $B / A=Q / A$. Hence $\operatorname{Ker}(g) \subseteq Q / A$ is a small submodule. Let, if possible, $C / A \subseteq \operatorname{Ker}(g)$ be a non zero AL-stable submodule of $Q / A$. Then $\operatorname{Ker}(f) \supset C \supset A$. Since $A$ is a maximal AL-stable submodule of $Q$ in $\operatorname{Ker}(f), C$ is not an AL-stable submodule of $Q$. Then by Lemma 3.3, $C / A$ is not an AL-stable submodule of $Q / A$. Therefore $g: Q / A \rightarrow M$ is an automorphism liftable cover of $M$.

From [15, Proposition 2.6], we have that any module has a quasi projective cover whenever, it has a projective cover. Then the following corollary follows from the above theorem.

Corollary 3.6. If a module $M$ has a projective cover, then it has an automorphism liftable cover.

In general, the converse of above corollary need not be true. With some certain condition as given in Theorem 3.7, it will be true.

Theorem 3.7. Let $\lambda: P \rightarrow M$ be an epimorphism from a projective module $P$ onto a module $M$. Then $M$ has a projective cover if and only if $P \oplus M$ has an automorphism liftable cover.

Proof. Suppose $M$ has a projective cover $p: P_{1} \rightarrow M$. Then $I_{P} \oplus p: P \oplus P_{1} \rightarrow$ $P \oplus M$ is a projective cover of $P \oplus M$. By Corollary 3.6, $P \oplus M$ has an automorphism liftable cover.

Conversely, assume that $P \oplus M$ has an automorphism liftable cover $\phi: L \rightarrow$ $P \oplus M$. Then $\phi \circ n: L \rightarrow P$ is an epimorphism, where $n$ is a natural projection of $P \oplus M$ to $P$. By the projectivity of $P$, we can view $P$ as a direct summand of $L$, i.e., $L=P \oplus L_{1}$ for some automorphism liftable module $L_{1}$. Then $\phi$ can be splitted as $\phi=I_{P} \oplus \phi^{\prime}$, where $\phi^{\prime}$ is the restriction of $\phi$ to $L_{1}$. Since $\operatorname{Ker}\left(\phi^{\prime}\right)$ is the homomorphic image of $\operatorname{Ker}(\phi), \operatorname{Ker}\left(\phi^{\prime}\right)$ is small in $L_{1}$. Hence $\phi^{\prime}: L_{1} \rightarrow M$ is an epimorphism with small kernel. 
By the projectivity of $P$, there exists a homomorphism $\lambda^{\prime}: P \rightarrow L_{1}$ such that $\phi^{\prime} \circ \lambda^{\prime}=\lambda$. Since $P \oplus L_{1}$ is automorphism liftable module, $P$ and $L_{1}$ are relatively projective to each other. Therefore the map $\lambda^{\prime}: P \rightarrow L_{1}$ splits, i.e., $L_{1}$ is a direct summand of $P$. Hence $\phi^{\prime}: L_{1} \rightarrow M$ is a projective cover of $M$.

Recall that a ring $R$ is said to be a (semi) perfect ring if every (finitely generated) module has a projective cover. In [5], Golan proved that a ring is perfect if and only if every module has a quasi projective cover. In [11], Selvaraj and Santhakumar characterized perfect ring by using dual automorphism invariant cover with certain condition. Also in [12], they proved that a ring is perfect if and only if every flat module is automorphism liftable. Here we characterized the same by using automorphism liftable cover.

Corollary 3.8. A ring $R$ is (semi) perfect if and only if every (finitely generated) module has an automorphism liftable cover.

Proof. Suppose every (finitely generated) module has an automorphism liftable cover. Since every (finitely generated) module can be written as an epimorphic image of a (finitely generated) projective module, by Theorem $3.7, R$ is a (semi) perfect ring.

Conversely, suppose $R$ is a (semi) perfect ring. Then by Corollary 3.6, every (finitely generated) module $M$ has an automorphism liftable cover.

In [6], Golan prove that a ring $R$ is semiperfect if and only if for all $n \geq 1$, every cyclic $R_{n}$-module has a quasi projective cover if and only if there exists an $n>1$ such that every cyclic $R_{n}$-module has a quasi projective cover. Inspired by this, we characterize the semiperfect ring by automorphism liftable covers.

Theorem 3.9. For any ring $R$, the following are equivalent:

(1) $R$ is semiperfect;

(2) For all $n \geq 1$, every cyclic $R_{n}$-module has an automorphism liftable cover;

(3) There exists an $n>1$ such that every cyclic $R_{n}$-module has an automorphism liftable cover.

Proof. (1) $\Rightarrow(2)$ It follows from the fact that if $R$ is semiperfect so is $R_{n}$ for all $n \geq 1$, by $[7$, Theorem 3$]$.

$(3) \Rightarrow(1)$ Let $n>1$ be satisfy the condition that every cyclic $R_{n}$-module has an automorphism liftable cover. Let $S$ be a right ideal of $R, S_{n}$ the right ideal of $R_{n}$ consisting of all matrices with entries from $S$. Let $e_{i j} \in R_{n}$ be the matrix with $1_{R}$ in the $(i, j)$ th position and zero else where. Then $R_{n} / e_{11} S_{n}$ is isomorphic to 
$P \oplus M$, where $M=e_{11} R_{n} / e_{11} S_{n}$ and $P=\sum_{i=2}^{n} e_{i i} R_{n} . P$ is clearly $R_{n}$-projective and the map $\lambda: P \rightarrow M$ which sends $\left[a_{i j}\right]$ to $e_{21}\left[a_{i j}\right]+e_{11} S_{n}$ is an $R_{n}$-epimorphism. Since $P \oplus M$ has an automorphism liftable cover over $R_{n}$, by Theorem 3.7, $M$ has a projective cover $\phi: Q \rightarrow M$ over $R_{n}$. Then $\phi\left(Q e_{11}\right)=(\phi Q) e_{11}=M e_{11}$ which is isomorphic, as an $R$-module, to $R / S$. Therefore $Q$ is $R_{n}$-projective and so $Q e_{11}$ is $R$-projective [7]. The induced $R$-homomorphism $\phi^{\prime}: Q e_{11} \rightarrow R / S$ is then a projective cover, proving (1).

$(2) \Rightarrow(3)$ is trivial.

In [10], Nguyen et al. proved that a $\operatorname{ring} R$ is semisimple Artinian if and only if every cyclic right module over $M_{n}(R), n \geq 2$ is a $D 3$-module. This result is also true when we replace $D 3$ by automorphism liftable.

Theorem 3.10. A ring $R$ is semisimple Artinian if and only if every cyclic right module over $M_{n}(R), n \geq 2$ is an automorphism liftable module.

Proof. From the proof of [10, Lemma 2.6.], we have a Morita equivalence between $R$ to $M_{n}(R)$ such that collection of all $n$-generated $R$-modules mapped onto collection of all cyclic $M_{n}(R)$-modules. Then by [12, Theorem 2.12], every $n$-generated $R$-module is automorphism liftable if and only if every cyclic module over $M_{n}(R)$, $n \geq 2$ is automorphism liftable. Hence by [12, Proposition 3.1], $R$ is semisimple Artinian if and only if every cyclic right module over $M_{n}(R), n \geq 2$ is an automorphism liftable module.

In [3], Byrd proved that a ring $R$ is uniserial if and only if every quasi projective module is quasi injective; equivalently if every quasi-injective module is quasiprojective. Here we characterize uniserial rings by using automorphism liftable and automorphism extendable modules.

Theorem 3.11. For any ring $R$, the following are equivalent:

(1) $R$ is uniserial;

(2) Every quasi projective module is automorphism extendable;

(3) Every quasi injective module is automorphism liftable;

(4) $R$ is quasi-Frobenius and every finitely generated quasi projective module is automorphism extendable;

(5) $R$ is quasi-Frobenius and every finitely generated quasi injective module is automorphism liftable.

Proof. $(1) \Rightarrow(2),(1) \Rightarrow(3),(1) \Rightarrow(4)$ and $(1) \Rightarrow(5)$ are follows from $[3$, Theorem $2.8]$. 
$(2) \Rightarrow(1)$ and $(3) \Rightarrow(1)$ : By [3, Theorem 2.8], it is enough to prove that every quasi (injective) projective module is quasi (projective) injective. Let $M$ be a quasi (injective) projective module. Then $M \oplus M$ is a quasi (injective) projective module. By hypothesis $M \oplus M$ is automorphism (liftable) extendable. Then by ([14, Lemma 7]) [12, Theorem 2.7], $M$ is quasi (projective) injective.

$(4) \Rightarrow(1)$ and $(5) \Rightarrow(1)$ are similar as above.

Acknowledgement. The author would like to express his indebtedness and gratitude to the referees for their helpful suggestions and valuable comments.

\section{References}

[1] A. N. Abyzov, T. C. Quynh and D. D. Tai, Dual automorphism-invariant modules over perfect rings, Sib. Math. J., 58(5) (2017), 743-751.

[2] A. N. Abyzov and C. Q. Truong, Lifting of automorphisms of factor modules, Comm. Algebra, 46(11) (2018), 5073-5082.

[3] K. A. Byrd, Some characterizations of uniserial rings, Math. Ann., 186 (1970), 163-170.

[4] L. Fuchs and K. M. Rangaswamy, Quasi-projective abelian groups, Bull. Soc. Math. France, 98 (1970), 5-8.

[5] J. S. Golan, Characterization of rings using quasiprojective modules, Israel J. Math., 8 (1970), 34-38.

[6] J. S. Golan, Characterization of rings using quasiprojective modules II, Proc. Amer. Math. Soc., 28(2) (1971), 337-343.

[7] S. M. Kaye, Ring theoretic properties of matrix rings, Canad. Math. Bull., 10 (1967), 365-374.

[8] M. T. Kosan, N. T. T. Ha and T. C. Quynh, Rings for which every cyclic module is dual automorphism-invariant, J. Algebra Appl., 15(5) (2016), 1650078 (11 pp).

[9] T. K. Lee and Y. Zhou, Modules which are invariant under automorphisms of their injective hulls, J. Algebra Appl., 12(2) (2013), 1250159 (9 pp).

[10] X. H. Nguyen, M. F. Yousif and Y. Zhou, Rings whose cyclics are D3-modules, J. Algebra Appl., 16(10) (2017), 1750184 (15 pp).

[11] C. Selvaraj and S. Santhakumar, A note on dual automorphism invariant modules, J. Algebra Appl., 16(2) (2017), 1750024 (11 pp).

[12] C. Selvaraj and S. Santhakumar, Automorphism liftable modules, Comment. Math. Univ. Carolin., 59(1) (2018), 35-44. 
[13] S. Singh and A. K. Srivastava, Dual automorphism-invariant modules, J. Algebra, 371 (2012), 262-275.

[14] A. A. Tuganbaev, Automorphisms of submodules and their extensions, Discrete Math. Appl., 23(1) (2013), 115-124.

[15] L. E. T. Wu and J. P. Jans, On quasi projectives, Illinois. J. Math., 11 (1967), 439-448.

\section{S. Santhakumar}

Department of Mathematics

Amrita School of Engineering - Coimbatore

Amrita Vishwa Vidyapeetham, India

https://orcid.org/0000-0003-4410-3696

e-mail: s_santhakumar@cb.amrita.edu 